\title{
Perfil sociodemográfico da mortalidade da população idosa no nordeste brasileiro
}

\author{
Sociodemographic profile of mortality in the elderly population in northeast Brazil
}

\author{
Bruna dos Reis Perreira ${ }^{a}$ \\ Orcid: https://orcid.org/0000-0002-5765-0341 \\ Ivia Mayana Oliveira de Jesus ${ }^{b}$ \\ Orcid: https://orcid.org/0000-0002-7548-0120 \\ Maísa Mônica Flores Martins ${ }^{\mathrm{c}}$ \\ Orcid: http://orcid.org/0000-0001-8329-614X
}

\begin{abstract}
Resumo
Introdução: $O$ envelhecimento populacional tem ocorrido de maneira radical e bastante acelerada principalmente nos países em desenvolvimento como o Brasil, esse processo de mudança do perfil demográfico representa um grande desafio para esses países, que precisam rapidamente inserir o tema do envelhecimento populacional na formulação de políticas públicas. Objetivo: Este estudo tem por objetivo caracterizar o perfil de mortalidade da população idosa do nordeste brasileiro. Materiais e Métodos: $\mathrm{O}$ presente projeto trata-se de um estudo ecológico, espaço-temporal, de caráter descritivo a partir de dados secundários de mortalidade da população idosa do nordeste brasileiro, no período de 2006 a 2016. As fontes de dados serão constituídas de elementos contidos nos diversos sistemas de informações disponíveis no Departamento de Informática do Sistema Único de Saúde. Resultados: Dos óbitos registrados, $45 \%$ eram de idosos longevos, as principais causas da mortalidade da população idosa decorrem principalmente por doenças do aparelho circulatório com destaque para as doenças cerebrovasculares com coeficiente de mortalidade para o sexo masculino de 505 por 100.000 homens e 401,3 por 100.000 mulheres, e outras causas como as neoplasias com maior coeficiente para a neoplasia da próstata e por seguinte as doenças endócrinas, nutricionais e metabólicas sendo diabetes mellitus a principal causa de morte, 268,4/ por 100.000 homens e 299,2 por 100.000 mulheres. Conclusão: Conclui-se que, essas informações são instrumentos imprescindíveis para o monitoramento das condições de vida e saúde, na orientação de estratégias de prevenção desses eventos, além de possibilitar o reconhecimento das demandas e padrões de serviços de saúde da população idosa para o planejamento, elaboração e avaliação de políticas de saúde.
\end{abstract}

Palavras-chave: idoso; indicadores de morbimortalidade; epidemiologia.

\begin{abstract}
Introduction: Population aging has occurred in a radical and quite accelerated manner, mainly in developing countries such as Brazil. This process of changing the demographic profile represents a major challenge for those countries that must involve population aging in public policies. Objective: This study aims to characterize the mortality profile of the elderly population in the Brazilian Northeast. Materials and Methods: This is an ecological, spatiotemporal, descriptive study based on secondary morbidity and mortality data from the Brazilian elderly population from 2006 to 2016. The data sources will be contained in the various information systems available in the Department of Informatics of the Unified Health System
\end{abstract}

\footnotetext{
${ }^{a}$ Graduanda do curso de enfermagem da Universidade Católica do Salvador, Brasil. E-mail: bruna.reis.pereira@hotmail.com

b Graduação em Enfermagem. Universidade Católica do Salvador, Brasil. E-mail: violiveira103@gmail.com

c Doutoranda em Saúde Pública. Universidade Católica do Salvador e Instituto de Saúde Coletiva da UFBA. E-mail: maisamonica@gmail.com
} 
(DATASUS). Results: Of the deaths recorded, 45\% were of long-lived elderly, the main causes of mortality in the elderly population are mainly due to diseases of the circulatory system, with an emphasis on cerebrovascular diseases with a mortality rate for men of 505 per 100,000 men and 401.3 per 100,000 women and other causes, such as neoplasms with a higher coefficient of prostate cancer and, later, endocrine, nutritional and metabolic diseases, with diabetes mellitus being the main cause of death 268.4 / per 100,000 men and 299.2 per 100,000 women . Conclusion: It is concluded that this information is an essential tool for monitoring health and living conditions, guiding strategies to prevent these events, and making it possible to recognize the demands and standards of the health services of the elderly population for planning elaboration and evaluation. health policies.

Keywords: aged; indicators of morbidity and mortality; epidemiology.

\section{Introdução}

O envelhecimento populacional tem ocorrido de maneira radical e bastante acelerada principalmente nos países em desenvolvimento como o Brasil, esse processo de mudança do perfil demográfico representa um grande desafio para esses países, que precisam rapidamente inserir o tema do envelhecimento populacional na formulação de políticas públicas e de pensar em soluções de reorganização social e de saúde suficientes para atender as necessidades dessa nova realidade populacional $^{1,2}$.

Estima-se que em 2025, existirá um total de aproximadamente 1,2 bilhão de pessoas com mais de 60 anos no mundo, no Brasil pode-se acompanhar uma tendência crescente do envelhecimento populacional, em 2012 a população com 60 anos ou mais era de 25,4 milhões, em cinco anos houve um crescimento de $18 \%$ atingindo a marca dos 30,2 milhões em $2017^{3}$.

Juntamente ao processo de transição demográfica, o padrão de adoecimento e morte da população também vem sofrendo transformações, o perfil epidemiológico resultado de uma série complexa de mudanças inter-relacionadas no comportamento de saúde e doença que ocorrem nas populações ao longo do tempo, são repercussões de um conjunto de transformações sociais, econômicas e principalmente demográficas ${ }^{4}$.

No Brasil, a transição epidemiológica ocorre de maneira "não convencional" aos países desenvolvidos, isso porque o contexto brasileiro demonstra cenários de reintrodução de processos infecciosos, permanência de endemias em algumas regiões do país, importantes variações geográficas quanto aos padrões epidemiológicos que demonstram regiões com condições de saúde comparáveis aos dos países desenvolvidos, e outras regiões com parâmetros de saúde e indicadores semelhantes a de países com os mais altos índices de pobreza (polarização epidemiológica), isso indica que não podemos afirmar que no Brasil ocorre uma transição epidemiológica clara, há uma sobreposição de diferentes condições epidemiológicas apresentadas ao longo dos $\operatorname{anos}^{5}$.

Quanto à tendência da morbimortalidade para essa população, as estimativas apontam que até 2020 é previsto que $46,4 \%$ de todas as mortes sejam atribuíveis a doenças cardiovasculares e expansão no número dos indivíduos com diabetes, passando de 51 milhões em 1995 para 72 milhões em 2025, já nos países em desenvolvimento a previsão é que a principal causa de morte e invalidez também seja por doenças cardiovasculares, com $71 \%$ das mortes por doenças isquêmicas do coração (DIC), 75\% das mortes por derrame e $70 \%$ das mortes por diabetes até o ano de $2020^{6}$.

Diante de tal situação é preciso encarar que para o Brasil e, especificamente, determinadas regiões do país que vivenciam um cenário de 
acentuada desigualdade social, pobreza, fragilidade das instituições e carência geral de recursos como, por exemplo, a região Nordeste, o envelhecimento da população é uma realidade que nos impõe um sentimento de preocupação.

Já que, essa região dispõe de características sociodemográficas desfavoráveis em relação às demais regiões, e que supostamente há peculiaridades quanto ao envelhecimento da população que não são conhecidas pela restrita quantidade de trabalhos sobre as condições de saúde dessa população, destacando que a maioria dos estudos sobre a temática se concentram em algumas capitais da região Sudeste, havendo poucas informações oriundas de outras regiões, como Nordeste 6,7 .

Desta forma, se faz necessário conhecer as principais causas de mortalidade que acometem a população idosa e caracterizar seu perfil sociodemográfico, já que essas informações são fundamentais para que possamos constatar as desigualdades dentro da faixa etária e da região de estudo, além disso, é possível traçar quais as principais demandas em saúde da população em estudo. Levando-se em consideração as questões apresentadas, o presente estudo irá caracterizar o perfil de mortalidade da população idosa do nordeste brasileiro, no período de 2006 a 2015.

\section{Metodologia}

Trata-se de um estudo ecológico, espaço-temporal, de caráter descritivo a partir de dados secundários de mortalidade da população idosa, no período de 2006 a 2015. A unidade de análise espacial é representada pelas Unidades Federativas da região do Nordeste brasileiro e os anos do período do estudo, a unidade temporal.

A fonte de dados constituiu-se de dados extraídos do Sistema de Informação sobre Mortalidade (SIM/SUS) a partir das declarações de óbitos. Essa, e outras bases de dados estão disponíveis no Departamento de Informática do Sistema Único de Saúde (DATASUS), em tempo real em uma plataforma virtual online ${ }^{8}$.

Para o cálculo das taxas de mortalidade, utilizou-se o número de óbitos disponíveis no SIM e os dados populacionais pelo censo 2010, do Instituto Brasileiro de Geografia e Estatística $(\mathrm{IBGE})^{9}$. A escolha e o tratamento das variáveis para análise no estudo foram baseados na disponibilidade das informações nos bancos de dados.

Para análise dos dados, foram consideradas como variáveis desfechos, as taxas de mortalidade da população idosa residente da Região do Nordeste brasileiro. Inicialmente, foi realizada análise absoluta e relativa entre os desfechos e as covariáveis do estudo. A tabulação dos dados foi realizada através do TabWin, o desenvolvimento das análises descritivas, os cálculos dos indicadores, além da construção dos gráficos e tabelas foram todos realizados no programa Excel for Windows 2013®. Como se trata de dados secundários de domínio público, a análise e aprovação de comitê de ética são dispensadas.

\section{Resultados}

Com base na análise absoluta das variáveis obtidas, verificou-se que 1.756.249 idosos foram a óbito na região Nordeste de 2006 a 2015, 45\% desses eram de idosos longevos ( 80 anos e mais) e $31 \%$ na faixa etária de 70 a 79 anos (Tabela 1). 
Tabela 1 - Distribuição das variáveis de caracterização sociodemográfica e de local de ocorrência dos óbitos da população idosa que foi a óbito no Nordeste brasileiro de 2006 a 2015.

\begin{tabular}{|c|c|c|c|c|}
\hline \multirow{2}{*}{ Variáveis } & \multirow{2}{*}{ Categorias } & 60 a 69 anos & 70 a 79 anos & 80 anos e mais \\
\hline & & $\mathrm{N}(\%)$ & $\mathrm{N}(\%)$ & $\mathrm{N}(\%)$ \\
\hline \multirow[b]{2}{*}{ Sexo } & Masculino & $242.887(57,35)$ & $285.677(52,78)$ & $359.977(45,48)$ \\
\hline & $\begin{array}{l}\text { Feminino } \\
\text { Ignorado }\end{array}$ & $\begin{array}{c}180.612(42,64) \\
36(0,01)\end{array}$ & $\begin{array}{c}255.514(47,21) \\
49(0,01)\end{array}$ & $\begin{array}{c}431.422(54,51) \\
75(0,01)\end{array}$ \\
\hline \multirow{6}{*}{ Cor/Raça } & Branca & $98.912(23,35)$ & $151.811(28,05)$ & $525.721(29,93)$ \\
\hline & Preta & $37.811(8,93)$ & $43.212(7,98)$ & $134.890(7,68)$ \\
\hline & Amarela & $1.242(0,29)$ & $1.791(0,33)$ & $5914(0,34)$ \\
\hline & Parda & $242.741(57,31)$ & $290.592(53,69)$ & $913.739(52,03)$ \\
\hline & Indígena & $696(0,16)$ & $871(0,16)$ & $2.967(0,17)$ \\
\hline & Ignorado & $42.133(9,95)$ & $52.963(9,79)$ & $173.018(9,85)$ \\
\hline \multirow{5}{*}{ Escolaridade } & Nenhuma & $112.578(26,58)$ & $179.412(33,15)$ & $325.293(41,10)$ \\
\hline & 1 a 3 anos & $93.968(22,19)$ & $116.981(21,61)$ & $150.268(18,99)$ \\
\hline & 4 a 7 anos & $51.050(12,05)$ & $53.443(9,87)$ & $60.687(7,67)$ \\
\hline & 8 a 11 anos & $28.556(6,74)$ & $25.340(4,68)$ & $28.218(3,54)$ \\
\hline & $\begin{array}{l}12 \text { anos e mais } \\
\text { Ignorado }\end{array}$ & $\begin{array}{c}14.230(3,36) \\
123.153(29,08)\end{array}$ & $\begin{array}{c}12.329(2,28) \\
153.735(28,40)\end{array}$ & $\begin{array}{c}13.119(1,66) \\
213.889(27,02)\end{array}$ \\
\hline \multirow{6}{*}{ Local ocorrência } & Hospital & $278.112(65,66)$ & $337.357(62,33)$ & $405.533(51,24)$ \\
\hline & Outro estabelecimento & $7.265(1,72)$ & $7.920(1,46)$ & $9199(1,16)$ \\
\hline & Domicílio & $115.472(27,26)$ & $179.297(33,13)$ & $362.246(45,77)$ \\
\hline & Via pública & $12.235(2,89)$ & $7.799(1,44)$ & $4.774(0,60)$ \\
\hline & Outros & $9.369(2,21)$ & $7.637(1,41)$ & $7.753(0,98)$ \\
\hline & Ignorado & $1.082(0,26)$ & $1.230(0,23)$ & $1.969(0,25)$ \\
\hline
\end{tabular}

Fonte: Sistema de Informações sobre Mortalidade - SIM

$\mathrm{Na}$ análise da variável sexo, observou-se que no somatório de todas as faixas etárias não houve grande predominância de um sexo para o outro, $50,6 \%$ para o sexo masculino e $49,4 \%$ do sexo feminino. Porém, quando a mesma variável foi analisada por faixa etária detalhada, foi constatado que há um predomínio de óbitos em indivíduos do sexo masculino na faixa etária dos "mais jovens" (60 a 69 anos), porém, existe uma inversão quando observa-se os longevos, nessa faixa etária as mulheres são a maioria (Tabela 1).

Quando a distribuição dos óbitos por raça/cor foi analisada, os idosos autodeclarados pardos são a maioria em todas as faixas etárias, sendo mais evidente nos idosos "mais jovens"; a segunda raça/cor mais predominante é a branca, dessa vez o número de óbitos é intensificado nos longevos, sendo possível observar um aumento de seis pontos percentuais dos idosos de cor branca da faixa etária dos "mais jovens" para os longevos (Tabela 1). Quanto à análise da escolaridade foi observado que obtivemos um quantitativo elevado de idosos com nenhuma escolaridade, ou com pouca permanência escolar (1 a 3 anos), a grande maioria apresentou nenhuma escolaridade (Tabela 1). 
Com relação ao local de ocorrência do óbito, predomina o ambiente hospitalar, principalmente nos idosos mais jovens, esse percentual diminui com o avançar da idade, na medida em que aumenta a faixa etária os óbitos nas residências tornam-se mais evidentes, com um impressionante acréscimo de dezoito pontos percentuais na frequência dos óbitos ocorridos no domicílio da faixa etária de 60 a 69 anos para 80 anos e mais (Tabela 1).

Foi analisado o estado civil de apenas três categorias (solteiro, casado e viúvo) dessa vez por sexo, nessa análise identificou-se que as mulheres idosas seguem suas vidas mais "solitárias" do que os homens. No sexo masculino, 59\% dos idosos que vieram a óbito no período de análise eram casados e, representando os menores percentuais, as categorias solteiro e viúvo com $20,7 \%$ e $20,4 \%$, respectivamente (Gráfico 1). Quando observamos o sexo feminino, há claramente uma discrepância dos valores em relação ao sexo oposto, a maioria das mulheres encontravam-se viúvas $(46,4 \%)$ e, por conseguinte, $27 \%$ delas eram solteiras (Gráfico 1).

Gráfico 1 - Caracterização da variável estado civil segundo sexo da população idosa que foi a óbito na Região Nordeste, no período de 2006 a 2015.

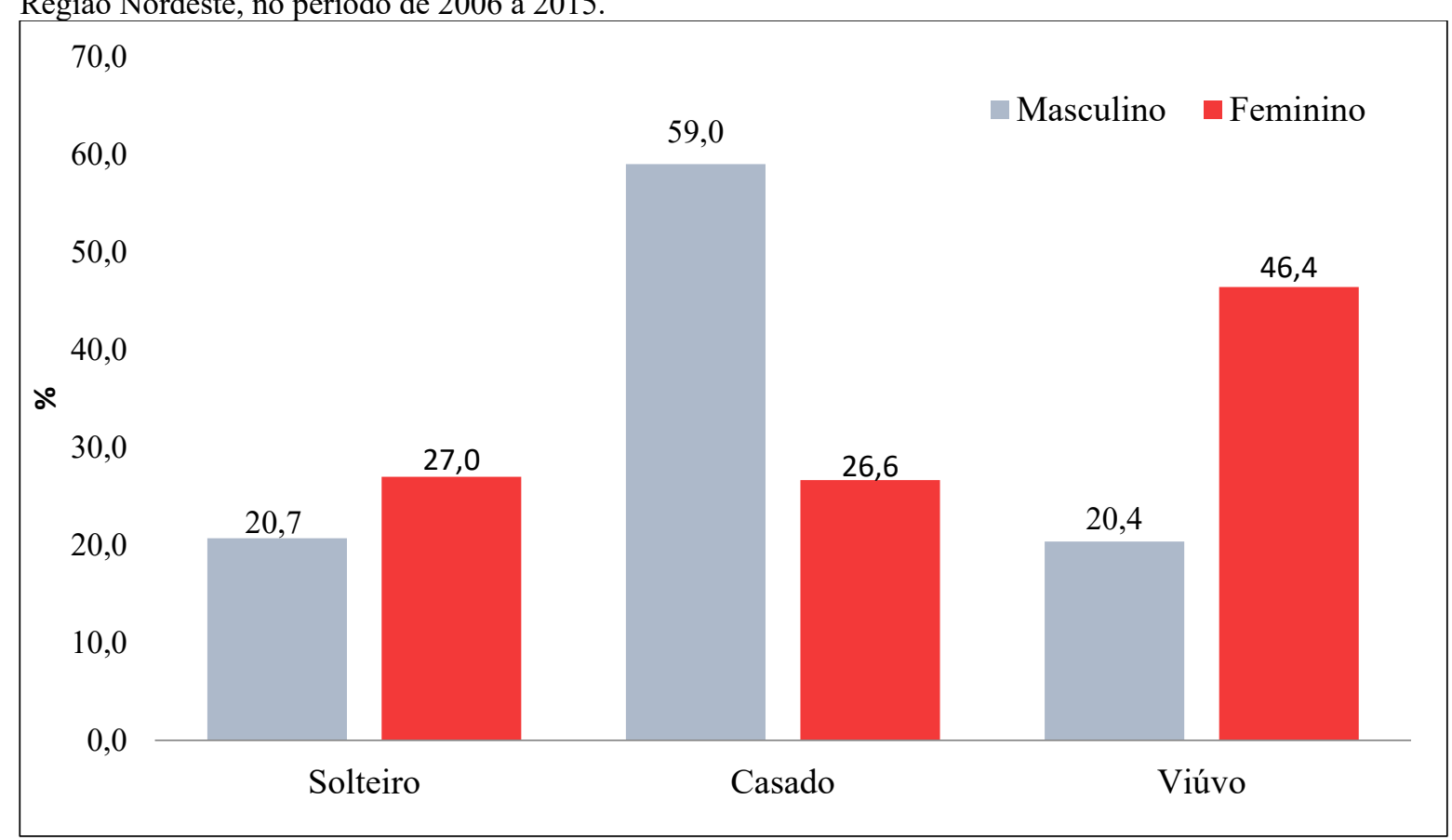

Fonte: Sistema de Informações sobre Mortalidade - SIM

Após o levantamento dos dados no Sistema de Informações sobre Mortalidade, foi possível constatar por análise das frequências, as principais causas de morte desses idosos por capítulos, segundo a Classificação Internacional de Doenças $10^{\mathrm{a}}$ Revisão (CID-10) categorizando por faixa etária (Gráfico 2).

De todos os capítulos da CID-10, sete de maior frequência foram selecionados, que somados representam $90,8 \%$ de todos os óbitos registrados no período de 2006 a 2015 (Gráfico 2).
Analisando esses capítulos por faixa etária, observa-se um comportamento diversificado em todas as idades, nitidamente as Doenças do Aparelho Circulatório (DAC) apresentam maior valor percentual em relação a todos os outros casos, sendo a primeira causa de óbito de todas as faixas etárias analisadas (Gráfico 2).

Nos longevos, as DAC possuem maior prevalência diante de todas as faixas etárias, a segunda causa de maior frequência são as Doenças do Aparelho 
Respiratório seguidas dos Sintomas e Sinais Anormais ao Exame Clínico e Laboratorial. Os idosos "mais jovens" detêm frequência elevada nos óbitos por DAC, sendo também a principal causa de morte desse grupo etário; as Neoplasias são a segunda maior causa de óbito dentre esses idosos, obtendo a maior frequência dentro deste capítulo. A segunda principal causa de óbito dos idosos de 70 a 79 anos são também as Neoplasias, seguidas das Doenças Endócrina Nutricionais e Metabólicas e posteriormente as Doenças do Aparelho Respiratório (Gráfico 2).

Gráfico 2 - Distribuição da mortalidade dos idosos pelos principais capítulos do CID-10 segundo a faixa etária na Região Nordeste, 2006 a 2015.



Fonte: Sistema de Informações sobre Mortalidade - SIM

Para entender melhor quais as causas específicas dentro dos grupos de maior frequência apresentados acima, além disso, identificar se há predominância de determinadas causas ligadas à questão de gênero, buscou-se construir o Coeficiente de Mortalidade Específica por Causa e Sexo (Tabela 2).

Tabela 2 - Coeficiente de mortalidade específica por causa e por sexo da população idosa da Região Nordeste, de 2006 a 2015.

\section{Causas de Óbito}

\begin{tabular}{cc}
\multicolumn{2}{c}{2006 a 2015} \\
Masculino & Feminin \\
& \\
505,0 & 401,3 \\
445,7 & 301,6 \\
381,7 & 255,7
\end{tabular}

151,0

57,8

32,9

301,6
75,2

445,7

-
40,5
26,6
25,5

Doenças isquêmicas do coração

Neoplasias

Neoplasia maligna da próstata

Neoplasias malignas da traqueia, brônquios e pulmões

Neoplasia maligna do estômago

Neoplasias malignas do fígado e vias biliares 


\begin{tabular}{lcc}
\hline \multicolumn{1}{c|}{ Causas de Óbito } & \multicolumn{2}{c}{2006 a 2015 } \\
\cline { 2 - 3 } & Masculino & Feminino \\
\hline Neoplasia maligna da mama & 1,1 & 43,7 \\
Doenças Endócrinas, Nutricionais e Metabólicas & & 299,2 \\
Diabetes mellitus & 268,4 & 30,9 \\
Desnutrição & 37,6 & 148,7 \\
Doenças do Aparelho Respiratório & & 101,8 \\
Pneumonia & 168,7 & 13,3 \\
Doenças crônicas das vias aéreas inferiores & 152,6 & 11,0 \\
Asma & & 151,5 \\
Sintomas, Sinais e Achados Anormais ao Exame Clínico e Laboratoriais & 21,4 \\
Morte sem assistência médica & 202,2 & 31,5 \\
Senilidade & 16,7 & 10,7 \\
Doenças do Aparelho Digestivo & & 4,0 \\
Doenças do fígado & 87,8 & 8,3 \\
Fibrose e cirrose do fígado & 27,3 & 29,6 \\
Doença alcoólica do fígado & 15,0 & \\
Úlcera gástrica, duodenal e péptica & & \\
\hline Fonte: Sistema de Informačos sobre Mortalicade- SIM. & \\
\hline
\end{tabular}

Fonte: Sistema de Informações sobre Mortalidade - SIM.

As três principais causas de mortalidade que apresentaram os maiores coeficientes foram as doenças cerebrovasculares (do sexo masculino 505 por 100.000 homens e para o sexo feminino 401,3 por 100.000 mulheres), seguidas das doenças isquêmicas do coração $(445,7 / 100.000$ para os homens e $301,6 / 100.000$ para as mulheres) e o infarto agudo do miocárdio $(381,7 / 100.000$ para os homens e 255,7/100.000 para as mulheres) percebe-se que os homens possuem os maiores coeficientes diante das Doenças do Aparelho Circulatório (Tabela 2).

No grupo das Neoplasias, destaca-se a neoplasia maligna da próstata, coeficiente expressivo que pode ser importante quando precisa-se buscar entender a gênese da mortalidade dos idosos do sexo masculino. Os demais coeficientes das Neoplasias para o sexo masculino apresentam-se em sua maioria mais elevados quando comparados ao sexo feminino, com exceção da neoplasia maligna da mama que representa um coeficiente de 43,7 óbitos a cada 100.000 mulheres e de 1,1 a cada 100.000 homens (Tabela 2).

Observa-se que o coeficiente das neoplasias malignas da traqueia, brônquios e pulmões e o coeficiente de neoplasia de estômago se mantêm mais elevados para o sexo masculino 75,2 e 57,8 por 100.000 homens respectivamente, enquanto que para o sexo oposto os coeficientes pelo mesmo agravo 40,5 é de 26,6 óbitos a cada 100.000 mulheres, respectivamente (Tabela 2).

Para as Doenças Endócrinas, Nutricionais e Metabólicas as duas principais causas são para diabetes mellitus e desnutrição, as mulheres possuem um maior coeficiente para o diabetes, porém, o coeficiente de mortalidade por desnutrição é maior nos homens. Fica claro que o sexo masculino possui altos índices de mortalidade na maioria das causas, entretanto, esse resultado não demonstra que a sobremortalidade dos homens idosos necessariamente se encontra nas doenças mais prevalentes de mortalidade, esses desfechos podem ser reflexos decorrentes de fatores comportamentais, ligados ao gênero (Tabela 2).

\section{Discussão}

Os resultados demonstrados neste estudo revelaram que a Região Nordeste sofre aumento anual constante e significativo das taxas de mortalidade no 
período analisado, principalmente para o grupo das Doenças e Agravos Não Transmissíveis (DANT), em que destacamse as Doenças do Aparelho Circulatório, Neoplasias e as Doenças do Aparelho Respiratório, acredita-se que esse perfil epidemiológico já é esperado em regiões cuja a população apresenta um processo de envelhecimento, as DANT e particularmente aquelas de natureza crônico-degenerativas são mais comuns em idosos, esse resultado é evidenciado em diferentes regiões do país e por diversos autores que se propõem a definir o perfil de morbimortalidade dessa população ${ }^{1,4,10,11}$.

Apesar de existir uma determinada semelhança em relação ao perfil epidemiológico encontrado em países mais desenvolvidos com elevados indicadores sociais como Noruega e Suíça, países que possuem Índices de Desenvolvimento Humano (IDH) muito alto, ou até mesmo em algumas regiões do país com melhores condições socioeconômicas como o Sul e o Sudeste brasileiro, não significa dizer que o Nordeste apresenta um processo de expansão da longevidade e de melhores condições de vida e saúde ${ }^{12}$.

Em estudo sobre carga global das doenças, o Nordeste apresentou maior carga para as doenças crônicas, por ser reflexo de más condições de vida e acesso a serviços de saúde para controle e manejo das doenças ${ }^{13}$. Além disso, apesar do fortalecimento da assistência farmacêutica, estudo demonstra que essa região apresentou diferença significativa em relação ao acesso aos medicamentos para doenças crônicas não transmissíveis ${ }^{14}$.

Isso porque ainda é possível encontrar um grande contingente de idosos que morrem sem nem mesmo terem um diagnóstico preciso, o que pode sugerir a deficiência dos serviços de saúde como a falta de assistência médica e baixa cobertura do SIM, além disso, desnutrição e mortes por doenças infecciosas e parasitárias que revelam condições sanitárias inadequadas não são indícios de uma sociedade modernizada, que oferece melhores condições de vida, acesso aos serviços de saúde, avanços nas tecnologias de saúde e prioriza ações preventivas ${ }^{4,15}$.

Mesmo que tenham ocorrido ao longo do tempo investimentos nas ações preventivas na região Nordeste, como aquelas oferecidas na Atenção Primária à Saúde (APS), ainda se faz necessária a expansão e maior investimentos desse modelo de atenção, visto que, as principais causas de morte aqui observadas poderiam ter sido evitadas caso houvesse uma maior cobertura e efetividade dos serviços da APS 7 .

É importante que as políticas de saúde para o envelhecimento sejam centradas na APS, visto que, os princípios determinados nesse nível de atenção são pautados na longitudinalidade, no vínculo e na responsabilização, além disso, as ações de prevenção de agravos, diagnóstico precoce, promoção da saúde, integração e assistência contínua são algumas das estratégias prioritárias desse nível de atenção que se tornam fundamentais a esse grupo etário, por possuir a particularidade de conseguir prevenir ou manejar determinadas condições incapacitantes e prevalentes de saúde na terceira idade $^{7,16,17,18}$. A melhor forma de combate é investir no fortalecimento do sistema de saúde, desde o financiamento, governança, gestão, recursos humanos em saúde, informação e acesso à tecnologia e medicações ${ }^{19}$.

Como foi analisado, os resultados relacionados ao coeficiente de mortalidade específica por causa e sexo, demonstram a heterogeneidade da mortalidade entre homens e mulheres, que em parte se deve às "questões de sexo" e às "questões de gênero", por exemplo, ao observar o coeficiente de mortalidade por neoplasia da próstata e dos demais coeficientes, que em sua maioria se mostraram mais elevados para o sexo masculino, percebe-se que, dentre múltiplos fatores, há evidências sobre a baixa sensibilidade e adesão da 
população masculina às campanhas preventivas e educativas relacionadas ao cuidado com a saúde, por outro lado, há também a escassez de políticas públicas específicas para a saúde do homem ${ }^{9}$.Vale ressaltar também, que a busca pelos serviços de saúde por parte da população masculina é bem reduzida por conta do machismo e horários de trabalho, sem contar com relutância em reconhecer que estão doentes ${ }^{20}$.

Existem outras questões como aquelas relacionadas aos comportamentos específicos do homem e da mulher, que dependem de fatores socioculturais que podem também estar atrelados a esses resultados, por exemplo, no Brasil o consumo de tabaco e álcool é superior entre os homens, logo, acredita-se que a sobremortalidade masculina oriunda das neoplasias malignas da traqueia, brônquios e pulmões, das doenças do fígado e suas complicações crônicas seja uma diferença associada a esses comportamentos de gênero, já que o hábito de fumar e beber faz parte de um estereótipo masculino influenciado por questões culturais e sociais $^{21}$.

É possível analisar o reflexo desse comportamento nos coeficientes de mortalidade por neoplasias, em que, por exemplo, nos valores dos coeficientes de mortalidade para neoplasia maligna do estômago é mais do que o dobro quando comparado ao sexo feminino, da mesma forma as demais neoplasias como as neoplasias malignas da traqueia, brônquios e pulmões e as neoplasias malignas do fígado e vias biliares também encontram-se mais elevadas para o sexo masculino.

De um lado, observamos uma maior mortalidade do sexo masculino, do outro os dados demonstram maior sobrevivência feminina ou morte precoce masculina, evidenciados pelos valores mais elevados dos óbitos desses na faixa etária de 60 a 69, os "mais jovens", fazendo com que os óbitos femininos se concentrem na faixa etária mais longeva, esses resultados retratam o fenômeno conhecido como feminização do envelhecimento, que está associado à maior longevidade das mulheres em comparação com os homens; consequentemente, é possível identificar maior presença relativa de mulheres na população idosa, principalmente nos estratos mais velhos ${ }^{4,10}$.

Podemos evidenciar esse fenômeno, observando o comportamento da variável "estado civil" apresentada neste estudo. É notório que há uma diferença significativa entre o estado de viuvez entre os idosos do sexo feminino e masculino, nota-se que as mulheres possuem o maior percentual na categoria viúvo, isso porque os homens morrem mais cedo $\mathrm{e}$ as mulheres sobrevivem por mais tempo, e acabam por morrerem viúvas.

A consequência desse fator na vida dos idosos é relativa, em um estudo comparativo foi identificado que nas mulheres a separação/divórcio ou a viuvez elevou a chance de morte, aquelas que se encontravam separadas e viúvas apresentaram taxas de mortalidade $82 \%$ e $35 \%$ maiores que a observada nas mulheres casadas, logo, à medida que a viuvez na vida de um idoso pode ser um acontecimento que configura grande sofrimento e fragilidade pela perda irreparável, pode ser que o mesmo fato possa representar um momento de aprendizagem e de possibilidades de desenvolvimento e adaptação em função das mudanças exigidas pela perda ${ }^{4}$.

Cada vez mais estudos demonstram que a feminização é um processo evidente quando se trata das alterações na estrutura etária $^{22}$. Porém, mesmo que esse processo possa remeter um triunfo à população feminina, não deve ser somente compreendida por essa perspectiva (dominância demográfica), mas também como um processo permeado por consequências da distribuição sexual do trabalho, em que a mulher ficou por muito tempo com a responsabilidade do cuidado domiciliar (como alimentação, vestimenta, saúde, etc.), um trabalho "invisível" e desvalorado pela sociedade, por privações 
de direitos como, baixa escolaridade, salários inferiores aos dos homens, violência e tantos outros fatores deletérios à sua qualidade de vida e sáude ${ }^{23}$.

A feminização pode também influenciar de forma positiva e/ou negativa tanto a sobrevida das mulheres quanto à instituição familiar, já que se sabe que tratase de uma questão de duplo risco social, ser mulher e idosa, coincidentemente o papel da mulher idosa como vínculo para a rede de apoio familiar também necessita de modificação $^{24}$. Por serem idosas e demandarem cuidado e serem responsabilizadas pelo cuidado, principalmente no ambiente doméstico ${ }^{23}$.

Assim como o Brasil, a região Nordeste experimenta, há algum tempo, rápido aumento da população idosa, o envelhecimento populacional tende a proporcionar, nas próximas décadas, desafios cada vez maiores às autoridades governamentais e especificamente no setor da saúde, o Nordeste apresenta um perfil epidemiológico complexo e cheio de adversidades, e de outros fatores que estão fortemente associados a condições de vida desfavoráveis que intensificam ainda mais a vulnerabilidade dessa população ${ }^{3}$.

Um fator importante na determinação do perfil de morbimortalidade de uma população é o seu nível educacional, quanto a esse fator podemos observar que estamos em grande desvantagem. Observase, diante da caracterização da variável escolaridade, que a região Nordeste apresenta um quantitativo elevado de idosos com nenhuma escolaridade, ou com pouca permanência escolar (1 a 3 anos), predominantemente na faixa etária do longevos.

Isso se deve ao fato que esses idosos não tiveram tantas oportunidades ao acesso escolar e nem a outras oportunidades educacionais. A escolaridade é apontada como um fator de proteção à saúde; indivíduos com melhores condições de educação tendem a ser mais responsáveis e conscientes com as questões relacionadas à saúde ${ }^{25}$.

Com referência à interpretação dos resultados dispostos no presente trabalho, vale ressaltar a existência de limitações quanto à utilização de dados secundários, já que a fonte de dados provém dos Sistemas de Informações em Saúde (SIS), assim sendo, admite-se a possibilidade da ocorrência de subnotificação e sub-registro dos óbitos, o que faz com que as informações obtidas não evidenciem seguramente a realidade e estejam dispostas a correções ${ }^{10}$.

\section{Conclusão}

Disparidades socioeconômicas e regionais são associadas às dificuldades normalmente enfrentadas pelo processo de envelhecimento, exacerbando as desigualdades pré-existentes. Além das desigualdades multifatoriais encontradas em todo território nacional, observa-se que, determinadas regiões, como por exemplo a Região Nordeste, representada neste estudo, sofrem com a intensificação da pobreza e das iniquidades sociais.

Por esses motivos, as regiões com acesso à educação, a melhores condições socioeconômicas e de acesso aos serviços de saúde, apresentam tendência decrescente na evolução da mortalidade, o que não foi constatado neste estudo, os resultados nos mostraram que a região Nordeste apresenta aumento no risco de morte para a população idosa ao decorrer dos anos.

Diante dos resultados encontrados no presente estudo, conclui-se que o grupo das Doenças do Aparelho Circulatório com destaque para as doenças cerebrovasculares e as isquêmicas do coração, Neoplasias, as Doenças do Aparelho Respiratório com maior percentual diagnóstico para a pneumonia e as doenças crônicas das vias aéreas inferiores e o alto índice de óbitos por diabetes e desnutrição, foram as principais causas de mortalidade nos idosos. 
Portanto, cabe ressaltar que as informações epidemiológicas e a caracterização sociodemográfica destacadas neste estudo são de suma importância e devem ser valorizadas por sua capacidade de alertar acerca dos riscos que imperam sobre a população idosa, principalmente pela sobrecarga das DANT que normalmente tornam-se crônicas e degenerativas, e de outros problemas e agravos à saúde como as doenças infecciosas e parasitárias e a desnutrição, que revelam o grau de privação socioeconômica e sanitária que tornam os idosos dessa região ainda mais vulneráveis e com mínimas chances de envelhecer ativamente e com qualidade de vida.

Logo, essas informações são instrumentos úteis para o monitoramento das condições de vida e saúde, na orientação de estratégias de prevenção desses eventos, de forma a interferir positivamente na história natural da doença, estabelecer diagnóstico precoce, prevenção de complicações das doenças crônicas, além de possibilitar o reconhecimento das demandas e necessidades de serviços de saúde da população idosa para o planejamento, elaboração e avaliação de políticas de saúde.

A construção de trabalhos como este, pode provocar espaços de discussão sobre a temática de forma a evidenciar a necessidade de ações públicas, mais respeito e maiores investimentos nos serviços de Atenção Básica, que são essenciais à população idosa por oferecer um modelo de saúde que transcende as características biológicas e as ações curativistas. Diante da perspectiva do cuidar integral e interdisciplinar garantem a atenção de todos os fatores envolvidos no perfil de saúde dos idosos.

\section{Referências}

1. Miranda GMD, Mendes ACG, Silva ALA. O envelhecimento populacional brasileiro: desafios e consequências sociais atuais e futuras. Rev. Brasileira de Geriatria e Gerontologia. 2016;19(3):507-19.

2. Veras R. Envelhecimento populacional contemporâneo: demandas, desafios e inovações. Revista Saúde Pública. 2009;43(3):548-54.

3. Borges GM, Campos, MBC, Silva, LGC. Transição da estrutura etária no Brasil: oportunidades e desafios para a sociedade nas próximas décadas. In: Ervatti LR, Borges GM, Jardim AP. (Org). Mudança Demográfica no Brasil no Início do Século XXI Subsídios para as projeções da população. Rio de Janeiro: Instituto Brasileiro de Geografia e Estatística - IBGE, 2015.

4. Medeiros WR. Mortalidade em idosos longevos e "mais jovens" no Brasil. 2015.108F. [Tese Doutorado em Saúde Coletiva] - Centro de Ciências da Saúde, Universidade Federal do Rio Grande do Norte, Natal, 2015.

5. Carvalho JAM, Garcia RA. O envelhecimento da população brasileira: um enfoque demográfico. Cad. Saúde Pública. 2003;19(3):725-733.

6. Coelho Filho JM, Ramos LR. Epidemiologia do envelhecimento no Nordeste do Brasil: Resultados de inquérito domiciliar. Revista de Saúde Pública. 1999;33(5):445453.

7. Lima-Costa MF, Barreto SM. Tipos de estudos epidemiológicos: conceitos básicos e aplicações na área do envelhecimento. Epidemiologia e Serviços de Saúde. 2003;12(4):189-201.

8. Ministério da Saúde (BR). DATASUS. Acessado em: 30 maio 2018.Disponível em: http://datasus.saude.gov.br/datasus.

9. IBGE. Instituto Brasileiro de Geografia e Estatística. Censo 2000 [Internet]. Rio de 
Janeiro: IBGE; 2014. Disponível em: http://www.ibge.gov.br. Acesso em: 22 maio 2018.

10. Virtuoso JF, Balbé GP, Mazo GZ, Pereira MGS, Santos FS. Morbidade e mortalidade da população idosa de Florianópolis: um estudo comparativo entre homens e mulheres. Revista Brasileira de Geriatria e Gerontologia. 2010;13(2)215-223. Disponível em: http://www.redalyc.org/pdf/4038/403838793006.pdf. Acesso em: 22 out. 2017.

11. Dantas I. C. et al. Perfil de morbimortalidade e os desafios para a atenção domiciliar do idoso brasileiro. Revista Kairós: Gerontologia. 2017; 20(1): 93-108. Disponível em: https://revistas.pucsp.br/index.php/kairos/article/view/2176-901X.2017v20i1p93108/22192. Acesso em: 18 de nov. 2017.

12. Oliveira PI. Brasil avança no IDH em 2019, mas terá novos desafios em 2020. Empresa Brasileira de Comunicação, 2019. Disponível em: http://agenciabrasil.ebc.com.br/direitos-humanos/noticia/2019-12/brasil-avanca-noidh-em-2019-mas-tera-novos-desafios. Acesso em: 19 de jan. 2020.

13. Leite CI, Valente GJ, Schramm JMA, Daumas RP, Rodrigues RN, Santos MF, Oliveira AF, Silva RS, Campos MR, Mota JC. Carga de doença no Brasil e suas regiões, 2008. Cad. Saúde Pública 2015; 31(7):1551- 1564.

14. Oliveira MA, Luiza VL, Tavares NUL, Mengue SS, Arrais PSD, Farias MR, et al. Acesso a medicamentos para doenças crônicas no Brasil: uma abordagem multidimensional. Rev Saúde Pública. 2016;50(supl 2):6s

Jorge MHPM, Dantas IC, Junior Pinto, EP, Medeiros KKAS, Souza EA. A mortalidade de idosos no Brasil: a questão das causas mal definidas. Epidemiol. Serv. Saúde. 2008;17(4): 2170-281. Disponível em: https://revistas.pucsp.br/index.php/kairos/article/view/2176-901X.2017v20i1p93108/22192. Acesso em: 22 out. 2017.

15. Lima-Costa MF, Matos DL, Camargos VP, Macinko, J. Tendências em dez anos das condições de saúde de idosos brasileiros: evidências da Pesquisa Nacional por Amostra de Domicílios (1998, 2003, 2008). Ciência \& saúde coletiva. 2011; 16(9):3689-3696. Disponível em: https://www.scielosp.org/pdf/csc/2011.v16n9/36893696/pt. Acesso em: 14 de nov. 2017.

16. Martins $\mathrm{AB}, \mathrm{D}$ 'Avila $\mathrm{OP}$, Hilgert JB, Hugo FN. Atenção Primária à Saúde voltada às necessidades dos idosos: da teoria à prática. Ciência \& Saúde Coletiva. 2014; 19(8):3403-3416. Disponível em: https://www.scielosp.org/pdf/csc/2014.v19n8/34033416/pt. Acesso em: 18 de nov. 2017.

17. Organização Mundial da Saúde (BR). Relatório Mundial de Saúde: atenção primária em saúde. Agora mais do que nunca. Brasília: OMS, 2008. Disponível em: http://www.who.int/eportuguese/publications/whr08_pr.pdf?ua=1. Acesso em: 10 out. 2017.

18. Malta DC, Bernal RTI, Lima MG, Araújo SSC, Silva MMA, Freitas MIF, et al. Doenças crônicas não transmissíveis e a utilização de serviços de saúde: análise da Pesquisa Nacional de Saúde no Brasil. Rev Saúde Pública. 2017;51 Supl 1:4s.

19. Filho ACAA, Almeida PD, Araújo AKL, et al. Perfil epidemiológico do diabetes mellitus em um estado do nordeste brasileiro. Rev Fund Care Online. 2017 jul/set; 9(3):641-647. DOI: http://dx.doi.org/10.9789/2175-5361.2017.v9i3.641-647.

20. F. Epidemiologia e o envelhecimento no Brasil. In: Freitas, EV. Tratado de geriatria e gerontologia. Rio de Janeiro: Guanabara Koogan, 2006. 
21. Tavares, F. D. C., \& Dátilo, G. M. P. D. A. Percepção da importância da participação de idosos em uma Universidade Aberta da Terceira Idade. Revista Equilíbrio Corporal e Saúde, 2012; 28-41.

22. Almeida AV, Mafra SCT, da Silva EP, Kanso SA. Feminização da Velhice: em foco as características socioeconômicas, pessoais e familiares das idosas e o risco social. Textos \& Contextos (Porto Alegre), 2015; 14(1): 115-131.

23. Lins I L, Andrade LVR. A feminização da velhice: representação e silenciamento de demandas nos processos conferencistas de mulheres e pessoas idosas. MediaçõesRevista de Ciências Sociais, 2018; 23(3): 436-465.

24. Mathias TAF, Aidar T. Diferencial de mortalidade na população idosa em um município da região Sul do Brasil, 1979-2004. Cien. Cuid. Saúde. 2010;9(1): 44-51. Disponível em: http://periodicos.uem.br/ojs/index.php/CiencCuidSaude/article/view/10529/5735. Acesso em: 18 de nov. 2017.

\section{Como citar este artigo:}

Perreira BR, Jesus IMO, Martins MMF. Perfil sociodemográfico da mortalidade da população idosa no nordeste brasileiro. Rev. Aten. Saúde. 2020; 18(64): 09-21. 